\title{
The Response of the Menstrual Cycle to Initiation of Hormonal Therapy in Transgender Men
}

Shazia Ahmad and Matthew Leinung*

\begin{abstract}
Purpose: Transition from female to male gender after initiation of hormonal therapy involves both phenotypic and physiological changes. The response to treatment can vary widely from person to person. In this study, we looked at the response of the menstrual cycle after the initiation of testosterone therapy and we wished to determine the time period and dose of testosterone leading to the cessation of menses in transgender men.

Methods: A retrospective chart review of 114 transgender men (individuals assigned female gender at birth but later identify themselves as males) seen for hormonal therapy in our transgender clinic from 2006 through 2016. Results: Forty patients were excluded from the study as they were either not menstruating (19), started therapy elsewhere (13), had incomplete data (6), or were treated with transdermal testosterone (2). Of the 74 patients begun on low dose (20-40 mg weekly) intramuscular testosterone, 55\% had cessation of menses within 6 months, 32\% took more than 6 to 12 months, and 7\% had not had complete cessation by 1 year and were prescribed progesterone ( $5 \%$ were given progesterone after menses had not ceased by 4 months for personal preference). The dose of testosterone correlated only loosely with the time of cessation (mean dose $40.1 \pm 8.1 \mathrm{mg} /$ week for those $<6$ months and $56.1 \pm 12.0 \mathrm{mg} /$ week for those who took 6-12 months).

Conclusion: The initiation of low to moderate testosterone was sufficient in leading to menstrual cessation in the majority of patients by 6 months and nearly all by 1 year. There was individual variability in the time to cessation with little correlation to testosterone dose.
\end{abstract}

Keywords: female to male transgender; menstrual cycles; testosterone

\section{Introduction}

Transgender individuals constitute up to $0.5 \%$ of general population. ${ }^{1,2}$ Increasing number of individuals are seeking hormonal treatment for gender dysphoria, and our experience is that transgender men (individuals assigned female gender at birth but later identify themselves as males) represent an increasing share of this population. ${ }^{4}$ In 2009, The Endocrine Society published clinical practice guidelines for the endocrine treatment of transgender persons. ${ }^{5}$ The ultimate goal of treatment is the overall well-being of the individual while achieving the transition to the desired gender. Hormonal therapy remains the central aspect of medical management. The initiation of hormonal therapy leads to both phenotypic and physiological changes that can take variable amounts of time. The secondary sexual characteristics of their birth-assigned gender, including ongoing menstrual cycles, are the source of a significant amount of psychological stress. Only a few studies in the literature have looked into the response of menstrual cycle to initiation of hormonal therapy. In 1986, Meyer et al. reported a longitudinal study of 30 transgender men, 14 of whom were studied for the response of the menstrual cycle to a fixed dose of testosterone cypionate $(200 \mathrm{mg}$ every 2 weeks). Menstruation ceased in six patients after the first dose, after the second dose in two patients, and by 4 months in the remaining six. ${ }^{6}$ In 2008, Moore et al. published an extensive review on management outcomes and adverse events in the transgender population, including their personal experience at Johns Hopkins Medical

Division of Endocrinology, Department of Medicine, Albany Medical College, Albany, New York.

*Address correspondence to: Mathew Leinung MD, FACE, Division of Endocrinology, Albany Medical College, 25 Hacket blvd, MC 141, Albany, NY 12208, E-mail: leinunm@mail.amc.edu

(C) Shazia Ahmad and Matthew Leinung 2017; Published by Mary Ann Liebert, Inc. This is an Open Access article distributed under the terms of the Creative Commons Attribution License, which permits unrestricted use, distribution, and reproduction in any medium, provided the original work is properly cited. 
Center. All transgender men achieved cessation of menses by the end of the study but no specific data were provided regarding the time required to achieve this endpoint. $^{7}$ In 2013, Nakamura et al. published data on 138 transgender men. The patients were treated at a gender identity clinic in Japan between 1997 and 2008. ${ }^{8}$ In this study, patients were divided into three groups based on the testosterone dose. The testosterone doses used in three groups were $250 \mathrm{mg}$ every 2 weeks, $250 \mathrm{mg}$ every 3 weeks, and $125 \mathrm{mg}$ every 2 weeks. The response to therapy was studied at 1 and 6 months. The authors concluded that although patients on higher dose testosterone showed early phenotypic changes at 1 month, however, at 6 months follow-up all patients achieved the same phenotypic and physiological changes. ${ }^{8}$ Another study by Pelusi et al. (2014) reported response of menstrual cycle in 45 transgender men who were randomly assigned to intramuscular (IM) testosterone $100 \mathrm{mg} / 10$ days, testosterone gel, or IM testosterone undecanoate. The author reported cessation of menses anytime between 30 and 41 weeks, with all transgender men achieving response by 1 year. ${ }^{9}$ In 2015 , Duetsch et al. reported the menstruation response to testosterone therapy in 31 transgender men. Patients were started on $50 \mathrm{mg} /$ week of IM testosterone and dose was increased to $70 \mathrm{mg} /$ week in 10 patients at 3 months due to persistent menstruation. This study reported cessation of menstruation in $84 \%$ (26 out of 31 ) transgender men at the end of 6 month duration. ${ }^{10}$

In this study, we looked at the time period between initiation of testosterone therapy and cessation of menstruation in our transgender male population followed at Albany Medical Center and determined the average weekly dose of IM testosterone needed for cessation.

\section{Methods}

This is a retrospective study of transgender men seen and followed at our Endocrinology clinic between 2006 and 2016. Approval for this study was obtained from the Albany Medical Center Institutional Review Board. Individual chart review was done and data were collected to calculate the time taken for the cessation of menstruation after the initiation of hormonal therapy. At each visit, patients were asked about their

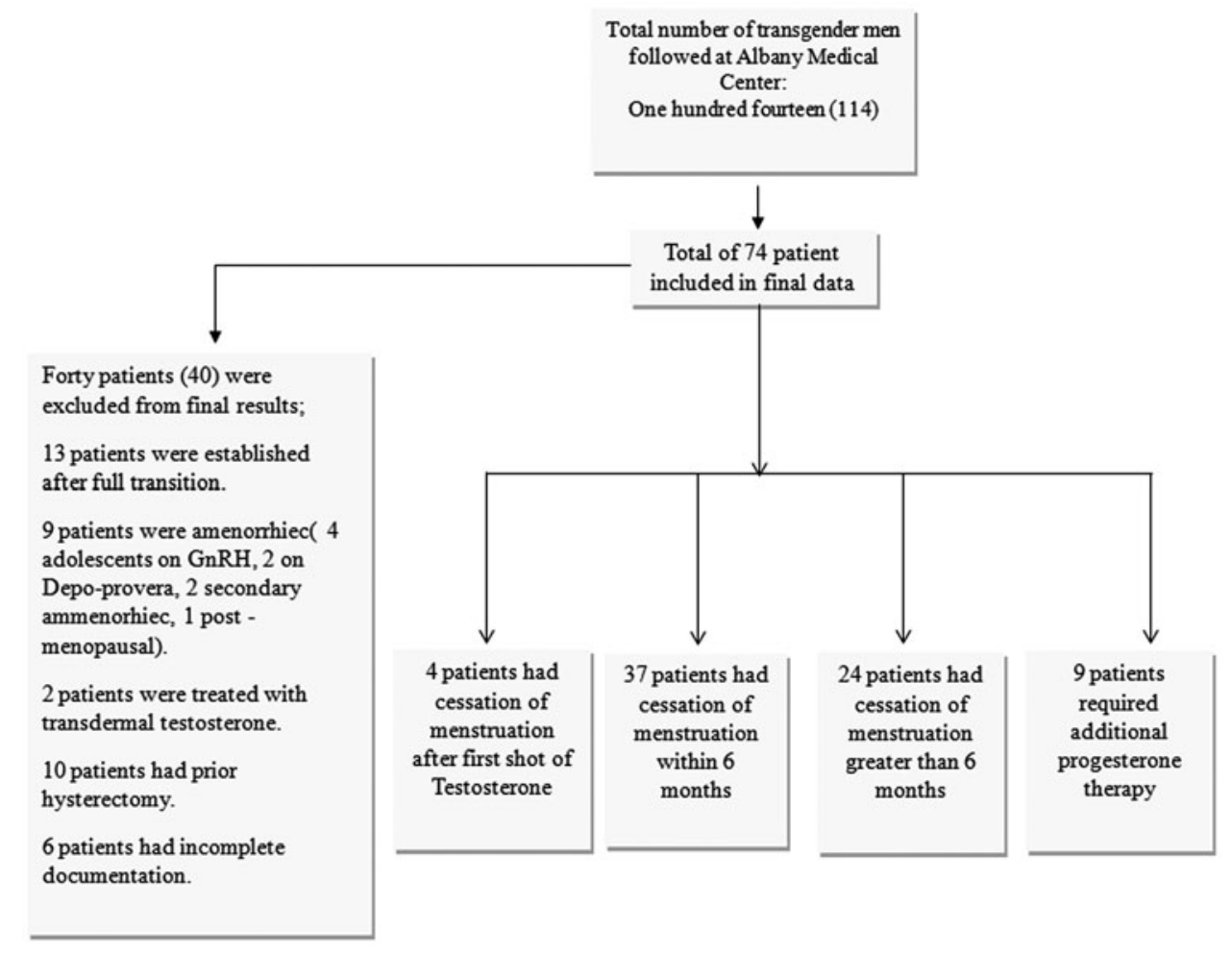

FIG. 1. Figure showing the total of 95 patients studied of which 35 patients were excluded from the final data collection. 
last menstrual history and testosterone dosing was documented. As per clinic protocol, any ongoing bleeding including intermittent spotting was documented by the provider. The charts that had no information about menses were excluded from final data collection. Cessation of menses was described as complete disappearance of menses, including intermittent spotting as confirmed from physician documentation. We also calculated the average weekly dose of testosterone taken at the time cessation occurred.

Our approach to the initiation of therapy is to begin with low dose (average $20-40 \mathrm{mg}$ weekly) and move to moderate dose ( $\sim 50-70 \mathrm{mg}$ weekly) in 3 to 9 months. Doses are individualized based on size, age, comorbid conditions, and patient concerns. Most patients move to full dose at 1 year (titrated to achieve normal age adjusted male levels).

\section{Results}

A total of 114 transgender men were seen from 2006 through 2016. Forty individuals were excluded from the study. Among those excluded, 13 had initiated treatment elsewhere, 10 had had prior hysterectomy (for reasons other than gender affirmation), 9 patients were amenorrheic (4 adolescents on pubertal suppression with GnRH analogues, 2 on Depo-Provera, 2 with secondary amenorrhea, and 1 postmenopausal), $6 \mathrm{had}$ incomplete data or follow-up, and 2 were treated with transdermal testosterone (Fig. 1).

Of the remaining 74 transgender men, 4 (5.4\%) had cessation of menses within 1 month after the first dose of IM testosterone injection, 37 patients (50\%) had cessation of menses between 2 and 6 months of treatment

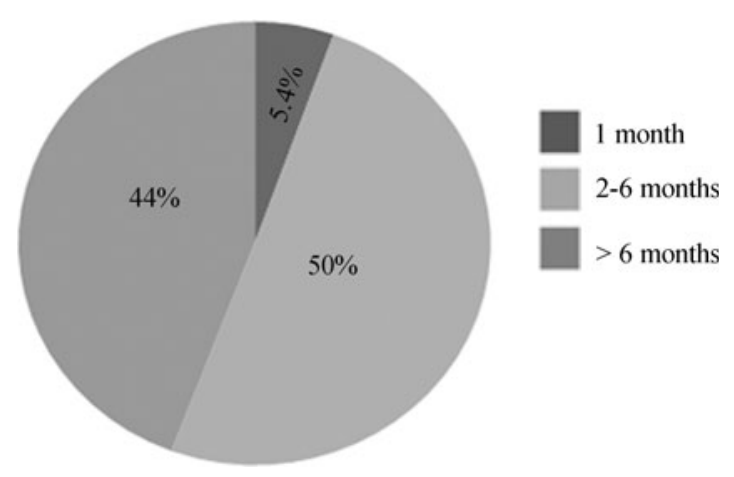

FIG. 2. Graph representing the percentage of patients who ceased menses within 1 month, 6 months, and $>6$ months.

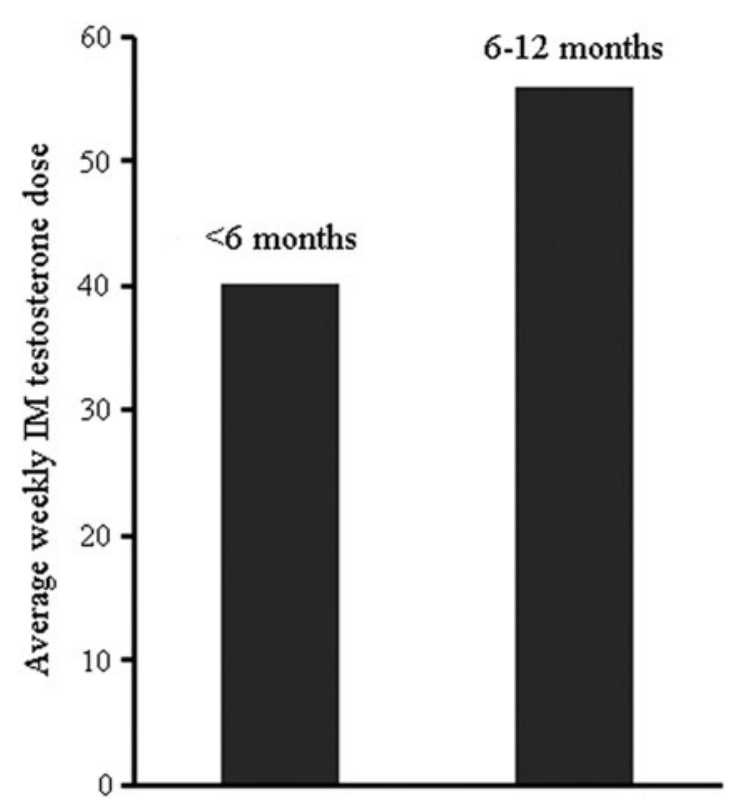

FIG. 3. Average weekly dose of IM testosterone dose in transgender men who ceased menses in $<6$ months ( $40.1 \pm 8.1 \mathrm{mg} /$ week) versus between 6 and 12 months ( $56.1 \pm 12.0 \mathrm{mg} /$ week). $(p<0.05) . \mathrm{IM}$, intramuscular.

(varying between 2 and 6 months), and 24 patients (32\%) took $>6$ months to achieve full cessation. Nine patients $(12 \%)$ were prescribed oral progesterone (five still menstruating at 1 year while on $50-60 \mathrm{mg} /$ week; four prescribed for patient preference) (Fig. 2). The doses of testosterone at the time of cessation of menses were analyzed to assess the effect of dose on cessation of menses. Individuals who ceased menses within 1 month of the first dose were found to be on an average of $40 \pm 8.1 \mathrm{mg}$ testosterone per week. For those whose cycles ceased between 2 and 6 months, the average calculated dose was $40.1 \pm 8.2 \mathrm{mg} /$ week, and for those who took $>6$ months, the average dose was also $56.1 \pm$ $12.0 \mathrm{mg} /$ week (Fig. 3). Those individuals who received progesterone for cessation of menses were on an average dose of $42.2 \pm 13.9 \mathrm{mg} /$ week.

\section{Discussion}

This study involves one of the largest cohorts in North America of transgender men treated over the past decade. We evaluated the response of the menstrual cycle to the initiation of therapy with low to moderate dose IM testosterone. We found that the cessation of menses occurred within 6 months in a majority of 
patients, with some transgender men being very sensitive to suppression with no further menstruation after the first injection. However, some patients responded slowly and required a longer duration of treatment and even additional therapy with progesterone. We found that the average weekly dose of testosterone administered to both groups (early and late responders) was statistically significant but clinically small ( $\sim 15 \mathrm{mg} /$ week).

Prior studies have reported the response of the menstrual cycle to testosterone therapy at various fixed dosages. ${ }^{6,8,9}$ We observed that the time to menstrual cessation was individualized, with only a small correlation to testosterone dose. Our results are similar to Nakamura et al., ${ }^{8}$ who reported that moderate dose (65 mg testosterone weekly) was able to induce suppression of menses in most patients. Similar findings were also reported by Deutsch et al. ${ }^{9}$ They reported cessation of menses within 6 months in $84 \%$ of patients with doses of 50-70 weekly.

This indicates that high testosterone doses are not typically necessary to suppress menstrual function. The Endocrine Society Guidelines recommend starting cross-sex hormone therapy in adolescents at low doses to lessen possible adverse results, but make no such specific recommendations in adults. ${ }^{5}$ Research documents a high rate of depression, anxiety, and suicidal ideation in transgender individuals than the general population. ${ }^{3,11}$ Although the overall benefits of hormonal therapy are clear, testosterone use has been associated with adverse effects on mood and behavior. ${ }^{12,13}$ With this in mind, we have routinely started hormone therapy at lower doses, but increasing more rapidly than when inducing puberty in adolescents. ${ }^{3}$ This approach appears to have worked well in that we have not experienced significant adverse mood or behavioral problems. Our results here show that low-to-moderate dose testosterone is able to induce menstrual suppression relatively quickly in a majority of transgender men.

We found individual variation in the susceptibility to menstrual suppression. At one extreme, some individuals had essentially immediate cessation upon initiation with an average dose of $40 \mathrm{mg} /$ week. This same dose was associated with suppression in 50\% of individuals overall by 6 months. Only slightly higher doses were able to induce amenorrhea in an additional $32 \%$ of individuals. Conversely, five patients (7\%) did not have suppression at doses of 50-60 mg/week. The reasons for this variability are unknown and will require further study.
A limitation of our study is the lack of a rigid protocol for testosterone dosing. However, since there has been one prescriber providing the majority of care for these individuals, we think the dosing has been fairly uniform.

In summary, we found that low-to-moderate dose testosterone therapy was able to suppress menstrual function in a majority of transgender men, but there was variability in the individual responses. The reasons for this variability are unknown and will require further study.

\section{Author Disclosure Statement}

No competing financial interests exist.

\section{References}

1. Tangpricha $V$, den Heijer M. Oestrogen and anti-androgen therapy for transgender women. Lancet Diabetes Endocrinol. 2017;5:291-300.

2. Shields JP, Cohen R, Glassman JR, et al. Estimating population size and demographic characteristics of lesbian, gay, bisexual, and transgender youth in middle school. J Adolesc Health. 2013;17:248-250.

3. Leinung MC, Urizar MF, Patel N, et al. Endocrine treatment of transsexual persons: extensive personal experience. Endocr Pract. 2013;19:644-650.

4. Ahmad S, Joseph J, Leinung MC. A cross-sectional study of clinical and psychosocial characteristics of a cohort of 371 transgender patient treated at Albany Medical Center. Endocrine Rev. 2016;37(2:Supplement): abstract FRI-139.

5. Hembree WC, Cohen-Kettenis P, Delemarre-van de Waal HA, et al. Endocrine treatment of transsexual persons: an Endocrine Society Clinical Practice Guideline. J Clin Endocrinol Metab. 2009;94:3132-3154.

6. Meyer W, Webb A, Stuart C, et al. Physical and hormonal evaluation of transsexual patients: a longitudinal study. Arch Sex Behav. 1986;15:121138.

7. Moore E, Wisniewski A, Dobs A. Endocrine treatment of transsexual people: a review of treatment regimens, outcomes, and adverse effects. J Clin Endocrinol Metab. 2003;88:3467-3473.

8. Nakamura A, et al. Dose-response analysis of testosterone replacement therapy in patients with female to male gender identity disorder. Endocr J. 2013;60:275-281.

9. Pelusi C, Costantino A, Martelli V, et al. Effects of three different testosterone formulations in female-to-male transsexual persons. J Sex Med. 2014;11:3002-3011.

10. Deutsch MB, Bhakri V, Kubicek K, et al. Effects of cross-sex hormone treatment on transgender women and men. Obstet Gynecol. 2015;125:605-610.

11. White HJM, Reisner SL. A systematic review of the effects of hormone therapy on psychological functioning and quality of life in transgender individuals. Transgend Health. 2016;1:21-31.

12. Bahrke MS, Yesalis CE, Wright JE. Psychological and behavioural effects of endogenous testosterone and anabolic-androgenic steroids. An update. Sports Med. 1996;22:367-390.

13. Mhillaj E, Morgese MG, Tucci $P$, et al. Effects of anabolic-androgens on brain reward function. Front Neurosci. 2015;9:295.

Cite this article as: Ahmad S, Leinung M (2017) The response of the menstrual cycle to initiation of hormonal therapy in transgender men, Transgender Health 2:1, 176-179, DOI: 10.1089/trgh.2017.0023.

$\mathrm{IM}=$ intramuscular 